\title{
Taking into Account Density Fluctuations in a Solvent in a Model of Dissolution
}

\author{
I. A. Ar'ev \\ Kyiv, Ukraine \\ Email: arev.igor@gmail.com
}

How to cite this paper: Ar'ev, I.A. (2019) Taking into Account Density Fluctuations in a Solvent in a Model of Dissolution. Open Journal of Physical Chemistry, 9, 204-215. https://doi.org/10.4236/ojpc.2019.94012

Received: July 2, 2019

Accepted: October 28, 2019

Published: October 31, 2019

Copyright (C 2019 by author(s) and Scientific Research Publishing Inc. This work is licensed under the Creative Commons Attribution International License (CC BY 4.0).

http://creativecommons.org/licenses/by/4.0/

\begin{abstract}
Earlier it was shown by different authors that there are cavities (vacancies, holes) in any liquid. The cavities should play a prominent role in dissolution processes. Nevertheless this fact was ignored in previous model of dissolution. The sizes of the cavities in different solvents containing benzene molecules were determined using solvent induced spectral shift method. The measurements of $S_{1} \leftarrow S_{0}$ benzene transition spectral shifts permit to conclude that 1) macroscopic excess volumes play an almost negligible role in processes of benzene dissolution in very different solvents and 2) the minimal size of the cavity in water able to accommodate benzene molecule coincides with the solute size. Generalization of this conclusion to other nonpolar aromatics leads to evaluation contraction of the solutes under aqueous solvent influence permits to predict the solubility values of other aromatics in water and to evaluate effect of enhancement hydrate cell around these molecules on solubility.
\end{abstract}

\section{Keywords}

Solubility, Solvent-Induced Spectral Shift, Microscopic Balance of Volumes, Fluctuation Cavities, Aqueous Solvent, Solute Contraction, Hydrate Shell Strengthening

\section{Introduction}

A question on quantitative prediction of solubility stands in front of scientists almost since ancient times. Nevertheless the first attempt to use quantitative parameters for qualitative prediction of solubility was done by Hildebrand in the middle of the twentieth century [1]. He introduced parameter named density of cohesion energy, $\delta=\sqrt{E / v}$, where $E$ equals to heat of evaporation and $v$ is molar volume. Good mutual solubility of two substances is predicted when their 
solubility parameters coincide. Hansen [2] improved the Hildebrand approach dividing parameter $\delta$ for components in accordance with interaction types. Both two approaches are able to predict if the solubility is good or poor, but they cannot give a quantitative answer to the question "how much is solubility?".

An attempt to answer this question was undertaken by Ben-Naim in the middle of the second half of the twentieth century [3]. According to Ben-Naim the dissolution process is realized according to the next manner. Firstly a cavity is created in a fixed position in the solvent. Then the solute which is in the fixed point in vacuum is transferred into the cavity. Finally the solute becomes free from the fixed place in the solvent. Sum of free energies of both two processes,

$$
\mu^{*}=G^{c}+G^{i}=-k_{B} T \ln c,
$$

is called pseudo chemical potential. It is not connected with any standard state. The term $G^{c}$ is free energy of creating the cavity and $G^{i}$ is free energy of interaction between the solute and the solvent. The term $c$ is concentration expressed in mole shares, $k_{B}$ is the Boltzmann constant, and $T$ is temperature.

This equation looks correct. Nevertheless successes of its direct application are very modest (look for example, ref. [4] and references therein). The cause is simple enough. Still between the first and the second world wars Frenkel and slightly later Schottky [5] proved that cavities (holes, vacancies) must exist even in the most ideal crystals. Naturally, they must exist in liquids. The fluctuations, namely fluctuation cavities, should participate in the process of dissolution. Luck [6] gathered a lot of indirect evidence that some kind of cavities really should exist in any liquid. A problem was how to measure these cavities, especially those of them which participate in the dissolution. The problem can be solved at least in part using the solvent induced spectral shift method. The method is in essence one of reverse spectroscopic problems when the shift of electronic spectrum of dissolved molecules serves a basis for decision of a question: how solvent molecules are distributed around the solute.

\section{The Solvent-Induced Spectral Shift}

\subsection{Model}

It is convenient to adopt the simplest model of the solution at least for a beginning. The solvent is considered as continual dielectric with dielectric constant $\mathcal{E}_{V}$ and refractive index $n$. The solute is represened by sphere of radius $r$ which is determined according to the Dejardin et al. procedure [7]: dependence should be built of molar volume of the solute substance in liquid state on its fluidity at different temperatures $V_{0}$, the volume at the fluidity equal to zero, is connected with $r$ by equation

$$
k_{p a c} r=\left(V_{0}\right)^{1 / 3}
$$


Here $k_{p a c}$ is packing factor. It equals to 1.88 for molecules whose shape does not sufficiently differ from spherical (Figure 1).

\subsection{Interactions in the Solution and the Spectral Shift}

There are two sorts of interactions in a dilute aqueous solution of nonpolar substance: solute-solvent and solvent-solvent interactions. Only the first one affects directly the spectral shift whereas the second of them affects indirectly participating in organization distribution solvent molecules around the solute. An electronic spectrum can be used for study the solution structure rather than vibrational one because in contrast to vibrational spectrum it belongs to whole molecule rather than to some of its fragments. Let the simplest case of the solute will be considered, when the solute is nonpolar molecule, and let electronic absorption of the solute is far from the solvent edge of the solvent absorption and let solute electronic states are mutually independent. Then the shift of a purely electronic or electronic-vibrational (vibronic) band in the transfer of a molecule from the gas phase to the solution may be considered as sum of the different contributions:

$$
\Delta v=\Delta v_{\text {disp }}+\Delta v_{\text {elst }}+\Delta v_{\text {chem }}
$$

Here $\Delta v$ is the shift of the spectral band expressed in wave numbers, $\Delta v_{\text {disp }}$ is contribution from dispersion interactions of the solute with solvent molecules, $\Delta v_{\text {elst }}$ is that part of the shift which is determined by interaction with sources of constant electric fields (ions, dipoles,...) in the solvent, and $\Delta v_{\text {chem }}$ is the term which corresponds to those chemical interactions including hydrogen bonding that do not change individuality of the solute molecule.

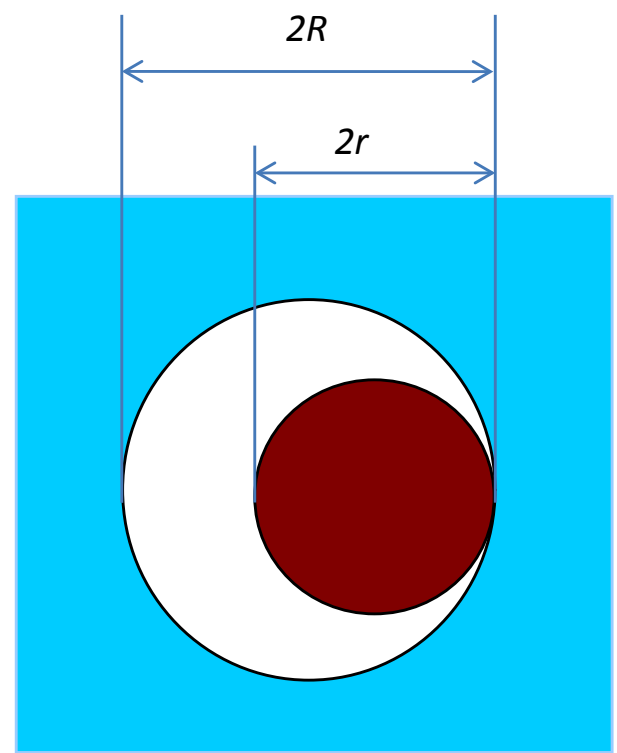

Figure 1. Model, A solute (sphere of radius $r$ ) in a spherical cavity of radius $R$ created in the solvent. 
Minimum of free energy of dispersion interaction take place when the solute touches the cavity border. The share of the shift stipulated by this type of interactions is

$$
\Delta v_{\text {disp }}=-C \varphi(R, r) f(n)
$$

where $C$ is a positive coefficient depending on properties of the transition in consideration, $\varphi(R, r)=R^{3} /\left[r^{3}(2 R-r)^{3}\right]$ is geometrical factor where $R$ is radius of the cavity containing the solute molecule whose radius is $r$, $f(n)=\left(n^{2}-1\right) /\left(n^{2}+2\right), n$ is refraction index [8].

It was shown in ref. [9] that there are neither electrical nor chemical interactions between the solute and the solvent in the aqueous solution of benzene. This fact will be used below at construction the simplest version of solubility model in which density fluctuations in the solute are taken into account.

This consideration is related to mutually independent electronic transitions. When electronic states are connected by a vibration, then the low which describes the shift suffers changes [10] [11]. So the shift of $S_{1}-S_{0}$ benzene transition is approximately expressed as

$$
\Delta v^{(10)}=-C_{1}\left|\Delta v_{10}\right|^{1.910}
$$

where $C_{1}$ is the positive coefficient, $\Delta v_{10}$ is the shift in the absence of electronic-vibrational coupling of the $S_{1}$ electronic state with other ones, and index 1.910 is a correction for this coupling [12].

Solvents whose aromatic molecules contain oxygen make exciplexes with high-energy states of aromatic solutes [12]. This fact permits to solve reverse spectroscopy problem using only the most low-energy transitions at handling with such solvents.

\subsection{Experimental Data}

Experimental details including purification of substances, recording and measuring spectral shifts were done in refs. [9] [10] [11]. Data concerning solubilities are cited below.

\section{Solubility}

\subsection{Microscopic Balance of Volumes}

The average size of cavities in the solvent able to participate in the dissolution process can be found from balance of volumes at dissolution:

$$
V_{1 u}^{v}=V_{a v}=V_{1 u}+V_{b v}+V^{E}
$$

Here $V_{1 u}^{v}$ is average volume of that cavity in the solvent, (superscript $v$ ) which contains one solute molecule (subscript $1 u$ ), $V_{a v}$ is the same volume in the solvent obtained after removal $a$ of its molecules, $V_{1 u}$ is average volume per one solute molecule in the solute substance, $V_{b v}$ is average volume of those fluctuating cavities in the solvent which participate in the dissolution process, and $V^{E}$ is excess volume of mixing the solution components. 
Solutions of benzene in different solvents can be considered as an instructive example. One obtains from Equation (5)

$$
\left|\Delta v_{\text {disp }}^{(10)}\right|^{1 / 910}=-k f(n) \varphi(R, r)
$$

where $k=2824.9695422 \mathrm{~cm}^{-1}$ and $r=2.72 \times 10^{-10} \mathrm{~m}$. The results of calculation microscopic balance of volumes at benzene dissolution in different solvents are listed in Table 1.

It is readily seen from the Table that benzene forms simple intermixing only with carbon tetrachloride, $R_{b v}=0$, not with all other considered solvents where $R_{b v}>0$. The positive values of $R_{b v}$ witness that just cavities participate in dissolution process, at least, in considered cases.

Next interesting consequence is seen from the Table. The excess volume plays negligible role In all considered cases. This fact supports conclusion made above that dissolution realizes through fluctuation cavities even in cases of infinite solubility, as for example in alkanes.

\subsection{Minimal Radius of the Suitable Cavity}

The minimal radius of the cavity yet participating in the process of benzene dissolution can be evaluated from the function of cavity size distribution in the solvent [5]:

Table 1. Solvents, their refractive indices, $n$, shifts of benzene $S_{1} \leftarrow S_{0}$ transition spectrum, $\Delta v^{(10)}$, average radii of cavities containing a benzene molecule, $R$, excess volumes of mixing, $V^{E}$, averaged over different sources, and $R_{b v}$ is radius of average fluctuation cavity participating in dissolution and $R_{b v}^{\prime}$ is the same without taking in consideration excess volume.

\begin{tabular}{|c|c|c|c|c|c|c|c|}
\hline Solvent & $n$ & $-\Delta v^{(10)}, \mathrm{cm}^{-1}$ & $R \times 10^{10}, \mathrm{~m}$ & $V^{E} \times 10^{30}, \mathrm{~m}^{3}$ & $R_{b v} \times 10^{10}, \mathrm{~m}$ & $R_{b v}^{\prime} \times 10^{10}, \mathrm{~m}$ & $R_{b v} / R_{b v}^{\prime}$ \\
\hline $\mathrm{CCl}_{4}$ & 1.4607 & 433 & 3.279 & 0.030037 & 0 & 0 & \\
\hline $\mathrm{CHCl}_{3}$ & 1.4459 & 386 & 3.329 & 1.4447 & 1.348 & 1.409 & 0.957 \\
\hline $\mathrm{H}_{2} \mathrm{O}$ & 1.33299 & 145 & 3.815 & -11.342 & 2.890 & 2.777 & 1.041 \\
\hline $\mathrm{D}_{2} \mathrm{O}$ & 1.33844 & 128 & 4.011 & -10.960 & 3.209 & 3.122 & 1.028 \\
\hline $\mathrm{CH}_{3} \mathrm{OH}$ & 1.3288 & 210 & 3.408 & 0.1214 & 1.760 & 1.764 & 0.998 \\
\hline $\mathrm{C}_{2} \mathrm{H}_{5} \mathrm{OH}$ & 1.3610 & 220 & 3.515 & 3.9448 & 2.032 & 2.105 & 0.965 \\
\hline $\mathrm{C}_{3} \mathrm{H}_{7} \mathrm{OH}$ & 1.3850 & 234 & 3.563 & -0.10793 & 2.235 & 2.216 & 1.009 \\
\hline$\left(\mathrm{CH}_{3}\right)_{2} \mathrm{SO}$ & 1.4770 & 381 & 3.431 & -3.8325 & 1.932 & 1.846 & 1.047 \\
\hline$c-\mathrm{C}_{6} \mathrm{H}_{12}$ & 1.4262 & 368 & 3.306 & 4.5826 & 1.054 & 1.237 & 0.860 \\
\hline$n-\mathrm{C}_{5} \mathrm{H}_{12}$ & 1.3575 & 218 & 3.508 & 0.7415 & 2.072 & 2.086 & 0.993 \\
\hline$n-\mathrm{C}_{6} \mathrm{H}_{14}$ & 1.3751 & 238 & 3.502 & 2.7433 & 2.016 & 2.069 & 0.974 \\
\hline$n-\mathrm{C}_{7} \mathrm{H}_{16}$ & 1.3876 & 246 & 3.524 & 4.5033 & 2.048 & 2.130 & 0.962 \\
\hline$n-\mathrm{C}_{8} \mathrm{H}_{18}$ & 1.3975 & 254 & 3.535 & 4.649 & 2.078 & 2.160 & 0.962 \\
\hline$n-\mathrm{C}_{10} \mathrm{H}_{22}$ & 1.4102 & 257 & 3.577 & 3.6221 & 2.211 & 2.268 & 0.975 \\
\hline$n-\mathrm{C}_{14} \mathrm{H}_{30}$ & 1.4293 & 270 & 3.601 & 4.2366 & 2.263 & 2.327 & 0.972 \\
\hline $1,4-\mathrm{C}_{4} \mathrm{O}_{2}$ & 1.4224 & 276 & 3.554 & -0.16771 & 2.127 & 2.128 & 1.000 \\
\hline
\end{tabular}




$$
\mathrm{d} p=\mathrm{d} R \operatorname{Rexp}\left[-G_{b v}^{c}(R) / k_{B} T\right]
$$

where $G_{b v}^{c}$ is average free energy of the fluctuation cavity surface which participate in dissolution (the same indexes are used in Equation (6)). It may be expressed through microscopic surface tension [13] [14]:

$$
G^{c}=\kappa \gamma \sigma \text {. }
$$

Here $\sigma$ is the cavity surface area, $\gamma$ is the macroscopic surface tension, and $\kappa$ is the coefficient correcting the macroscopic surface tension to the microscopic one. It is expressed as [13]:

$$
\kappa \cong 1+\left(\sigma_{1} / \sigma\right)\left(\kappa_{1}-1\right)
$$

where $\sigma_{1}$ is the area of the surface of the cavity created in the liquid as a result of removal of one of its molecules, and

$$
\kappa_{1} \cong\left(\gamma \sigma_{1}\right)^{-1} k_{B} T \ln \left(k_{B} T / P_{s} V_{1}\right)
$$

Here $P_{s}$ is pressure of saturated vapor and $V_{1}$ is the volume per one molecule in the liquid. For associated liquids

$$
\kappa_{1} \cong\left(\gamma \sigma_{1}\right)^{-1} k_{B} T \ln \left(k_{B} T / \xi P_{s} V_{1}\right)
$$

where $\xi$ is average degree of association of vapor molecules [15].

Now $V_{b v}$ can be expressed as

$$
\left(R_{b v}\right)^{2}=\frac{\int_{R_{m}}^{\infty} \mathrm{d} R R^{3} \exp \left[-G^{c}(R) / k_{B} T\right]}{\int_{R_{m}}^{\infty} \mathrm{d} R R \exp \left[-G^{c}(R) / k_{B} T\right]}=\left(R_{m}\right)^{2}+\frac{k_{B} T}{4 \pi \kappa \gamma}
$$

Here $R_{m}$ is the radius of that minimum cavity which is still good for acceptance the solute, $\gamma=71.95 \mathrm{mN} / \mathrm{m} \quad[16], \quad \kappa \approx 1 \quad[15]$. Hence

$R_{m}=2.81 \times 10^{-10} \mathrm{~m}$. This value exceeds $r=2.72 \times 10^{-10} \mathrm{~m}$ [7] adopted here for benzene molecular radius only about $3 \%$. This is too low difference for our crude model of solution. Therefore we may think that the minimal size of the cavity in water able to take the benzene molecule coincides with benzene molecule size. This conclusion is extended further to other big nonpolar solutes.

\subsection{Approaches to Solubility}

\subsubsection{Fluctuation Approach}

Let us consider low solubility of substance consisting of big nonpolar molecules, so low solubility that solute molecules do not touch each other. Let $N$ is full amount of solvent molecules and $n$ is amount of cavities able to accept a solute molecule. Then solubility is determined by

$$
\mu^{*} / k T=-\ln (n / N)=-\ln c
$$

If the similar coincidence takes place also for other nonpolar big rigid nonpolar molecules, one has 


$$
\ln \left(n_{e} / n\right)=\ln \frac{\int_{r_{e}}^{\infty} \mathrm{d} R R \exp \left(-4 \pi \gamma R^{2} / k_{B} T\right)}{\int_{r}^{\infty} \mathrm{d} R R \exp \left(-4 \pi \gamma R^{2} / k_{B} T\right)}=4 \pi \gamma\left(r_{e}^{2}-r^{2}\right) / k_{B} T .
$$

One obtains after substituting the right side of Equation (15) into Equation (14):

$$
\mu^{* \prime}=\mu_{e}^{*}+4 \pi \gamma\left(r^{2}-r_{e}^{2}\right)
$$

Here prim numbers the approach to evaluation the solubility. The results obtained with this approach are given in the third column of Table 2. They are not very significantly deviated from empirical data. Note that this approach is not connected even with phase states of solution components.

\subsubsection{Energetic Approach}

Free energy of molecular transfer out of the fixed position in the substance which will be dissolved into the fixed position in vacuum and then into the fixed position in the solvent, $\mu^{* \prime \prime}$, is considered in the second approach called energetic one. In the idealized case when solute properties do not change at these transitions,

$$
\mu^{* \prime \prime}=G_{1 u}^{i v}+G_{1 u}^{c v}-G_{1 u}^{i}-G_{1 u}^{c}
$$

Here $G_{1 u}^{i v}$ is free energy of interaction (superscript $i$ ) of one solute molecule (subscript $1 u$ ) with the solvent (superscript $v$ ), $G_{1 u}^{c v}$ is free energy of creation the cavity in the solvent (superscript $c$ ) where the solute molecule can be placed, $G_{1 u}^{i}$ is free energy of interaction between the solute and its environment in the solute substance, and $G_{1 u}^{c}$ is free energy of creation the cavity instead removed solute molecule. The detailed Equation (17) looks as

Table 2. Geometrical characteristics, quasi-chemical potentials of dissolution and in-

\begin{tabular}{|c|c|c|c|c|c|c|c|}
\hline \multirow{3}{*}{ Solute } & \multirow{3}{*}{$\begin{array}{c}10^{10} r \\
\mathrm{~m}\end{array}$} & \multicolumn{4}{|c|}{$\mu / k T$} & \multirow{3}{*}{$\begin{array}{c}10^{10} \delta r \\
\mathrm{~m}\end{array}$} & \multirow{3}{*}{$\Delta N_{1}$} \\
\hline & & \multicolumn{3}{|c|}{ Calculation } & \multirow{2}{*}{ Experiment } & & \\
\hline & & Equation (16) & Equation (22) & Equation (32) & & & \\
\hline benzene & 2.72 & - & 7.91 & - & 7.85 & - & 0 \\
\hline toluene & 2.895 & 9.85 & 8.97 & 9.41 & 9.16 & -0.04 & 3.6 \\
\hline o-xylene & 3.045 & 12.87 & 10.34 & 11.10 & 10.33 & -0.06 & 7.1 \\
\hline p-xylene & 3.055 & 12.01 & 10.03 & 11.02 & 10.31 & -0.08 & 7.4 \\
\hline m-xylene & 3.055 & 12.01 & 10.10 & 11.06 & 10.33 & -0.07 & 7.4 \\
\hline ethylbenzene & 3.04 & 11.80 & 10.00 & 10.90 & 10.26 & -0.07 & 7.0 \\
\hline cumene & 3.19 & 13.91 & 10.56 & 12.24 & 11.35 & -0.12 & 10.7 \\
\hline styrene & 2.97 & 10.85 & 10.51 & 10.68 & 9.86 & -0.01 & 5.4 \\
\hline naphthalene & 3.054 & 12.71 & 12.60 & 12.66 & 12.31 & $-1 \cdot 10^{-5}$ & 6.4 \\
\hline
\end{tabular}
crease of aqueous molecules in the first hydrate cell of some substituted benzenes in water at $293 \mathrm{~K}$. 


$$
\mu^{* \prime \prime}=G_{a v}^{i}+\Delta G^{i}+G_{a v}^{c}-G_{b v}^{i}-G_{b v}^{c}-G_{1 u}^{i}-G_{1 u}^{c}
$$

where $G_{a v}^{i}$ is free energy of interaction between content of sphere which radius is $R_{1 u}^{v}$ before removal a solvent molecules out of it and the rest solvent, $G_{a v}^{c}$ is free energy of this cavity surface, terms with subscripts $b v$ and $1 u$ describe similar characteristics of original vacancies in the solvent and components of that free energy which must be spend for removal one molecule out of the substance to be dissolved, respectively, and $\Delta G^{i}$ is a correction which must be introduced into the process description after replacement claster of a solvent molecules for one solute molecule. According to [13] [14],

$$
G^{i}=-2 G^{c}
$$

and Equation (18) becomes simplified to

$$
\mu^{* \prime \prime}=-G_{a v}^{c}+G_{b v}^{c}+\Delta G^{i}+G_{1 u}^{c}
$$

When size of the cavity which contains the solute molecule is held rather by induced electrostatic forces than by collisions at thermal movement, then equilibrium takes place at

$$
-G_{a v}^{c}+G_{b v}^{c}+\Delta G^{i}=0
$$

Then

$$
\mu^{* \prime \prime}=G_{1 u}^{c}
$$

In essence, $\mu^{* \prime \prime}$ is pseudo chemical potential of transfere a molecule out of the condensed substance liable to dissolution into the solvent. It is described with the next equation:

$$
\mu^{* \prime \prime} / k T=-\ln C
$$

Thus it is the quasi chemical potential. The double prim numbers the approach to evaluation $\mu^{*}$. The data on calculated solubilities are given in the fourth column of Table 2 . They also are close enough to empirical results.

When the solute molecule is transferred out of a solid phase into the solvent then Equation (23) should be specified. Zhang and Gobas [17] supposed that a surface molecule of a solid substance dissolving in a liquid is bound with other ones in that manner as molecules of super cooled liquid. Then

$$
\mu^{* \prime \prime}=k T \ln \left(R T / p_{s} V_{1 u}\right)-\Delta G_{f}
$$

Here $\Delta G_{f}$ is a change of free energy at conversation the solute substance into the state of super cooled liquid which equals to

$$
\Delta G_{f}=\Delta H_{f}\left(1-T / T_{m}\right)+\int_{T}^{T_{m}} \Delta C_{p} \mathrm{~d} T-T \int_{T}^{T_{m}} \Delta C_{p} \frac{\mathrm{d} T}{T}
$$

Here $\Delta H_{f}$ is enthalpy of solute substance fusion at melting point $T_{m}$ and $\Delta C_{p}$ is change of specific heat at changing the phase state. For example, Eq. (25) may be used for evaluation that characteristic of naphthalene which is solid at the room temperature. One can evaluate function $\Delta C_{p}(T)$ applying 
square-law extrapolation specific heat of the liquid phase taking necessary values from ref. [18]: $\Delta C_{p}=4.184\left(1.00286 \times 10^{-4} T^{2}-2.2563 T+66.1362\right) \mathrm{J} / \mathrm{mol} \cdot \mathrm{K}$.

\subsubsection{United Approach}

Corrections to solute size changes should be introduced in both two above described approaches. Correcting term $\delta \mu^{\prime}$ to the quasi-chemical potential $\mu^{* \prime}$ is caused by the molecular size decreasing because of pressing by reaction field forces [9]. It can be found from the next expression:

$$
\begin{aligned}
\mu^{* \prime} & =\mu_{e}^{* \prime}+\delta \mu^{\prime} \\
& =\mu_{e}^{* \prime}+4 \pi\left[(r+\delta r)^{2}-r_{e}^{2}\right] \gamma \\
& \cong \mu_{e}^{* \prime}+4 \pi\left(r^{2}+2 r \delta r-r_{e}^{2}\right) \gamma
\end{aligned}
$$

and

$$
\delta \mu^{\prime} \simeq 8 \pi \gamma r \delta r .
$$

Such correction is the negative value because its sign coincides with the sign of $\delta r$.

Correction to $\mu^{\prime \prime}$ in energetic approach looks as

$$
\mu^{\prime \prime}=\mu^{* \prime \prime}+\delta \mu^{* \prime \prime}
$$

where $\delta \mu^{* \prime \prime}$ is correction which takes into account reversible positive work making by forces of hydrophobic (electric) repulsion which compress the solute molecule.

$$
\delta \mu^{* \prime \prime} \approx 4 \pi \gamma\left(R_{1}^{2}-R^{2}\right)
$$

Here $R_{1}$ is radius of the cavity containing the solute in the case if it is not subjected to deformation, and $R$ is the same after deformation. $R$ can be expressed in quasi-spherical approximation as $R=\left[R_{1}^{3}-r^{3}+(r+\delta r)^{3}\right]^{1 / 3}$. We get after expansion $R$ in the Tailor series and taking into account that $R \approx 3 r / 2$, and neglecting the infinitesimal terms of decomposition, that

$$
\delta \mu^{* \prime \prime} \approx-8 \pi \gamma r \delta r
$$

One obtains comparing Equations. (27) and (30) that

$$
\delta \mu^{* \prime}=-\delta \mu^{* \prime \prime}
$$

It follows from Equation (31) that difference between values $\mu^{* \prime}$ and $\mu^{* \prime \prime}$ is caused only by contraction of substituent size under reaction (reactive field) of the aqueous solvent on the solute. Hence

$$
\mu^{*}=\left(\mu^{* \prime}+\mu^{* \prime \prime}\right) / 2
$$

Only deformation of the solute is taken into consideration at calculating $\mu^{*}$. The value of the solute size contraction is

$$
\delta r \approx-\Delta \mu /(16 \pi \gamma r)
$$


where $\Delta \mu=\mu^{* \prime}-\mu^{* \prime \prime}$.

The values of $\mu^{*}$ with values of $\delta r$ are given in the fifth column of Table 2. Firstly, it is readily seen from the Table that the predicted values of $\mu^{*}$ are closer to the measured ones then predicted by any of above approaches. So the main points of presented consideration look right. Secondly, the substance with rigid molecules, namely naphthalene, does not show any size contraction under aqueous solvent influence. Contractions show molecules containing alkyl substituents. The more branched is a substituent, the more is contraction. This fact is evidently caused by facility of ordinary bond deformation.

\subsubsection{Taking into Account Solvent Shell Strengthening}

Interaction between water molecules in hydrate shell of benzene molecule is more strong then in pure water. Really, ions $\mathrm{K}^{+}$and $\mathrm{Cl}^{-}$destroy water structure, i.e., weaken interaction between the molecule and other water molecules [19]. Nevertheless, addition salt $\mathrm{KCl}$ into aqueous solution of benzene does not destroys its hydrate cell, in contrast to addition such salts as $\mathrm{RbCl}$ and $\mathrm{CsCl}$ [9] which are more actively then $\mathrm{KCl}[19]$.

One can evaluate contribution of this enhancement into the $\mu^{*}$ value comparing calculated values with measured ones. The corresponding correction equals to

$$
\Delta \mu^{*} / k T=-0.214 \Delta N_{1}+0.055
$$

Here $N_{1}$ is amount of water molecules in the first hydrate shell of benzene and $\Delta N_{1}$ is the change of this amount after transition to another solute. Approximately

$$
N_{1}=4 \pi\left[R+\left(V_{1 u}\right)^{1 / 3} / 2\right]^{2} /\left(V_{1 u}\right)^{2 / 3},
$$

where $R \approx 1.5 r$. The correlation factor of dependence (34) equals to $\rho=0.943$, of root mean square deviation of coefficient at $\Delta N_{1} \sigma_{A}=0.080$ and of free term $\sigma_{B}=0.028$. One can readily see from Equation (34) that interaction between water molecules in the first hydrate shell is enhanced owing to interaction with the solute and lowering the solvent free energy per one its molecule in the solvent shell equals approximately $0.2 k T$. The low value of the free term in the right side of Equation (34) witness that adopted approximation is correct (see Table 3).

\section{Conclusions}

The above consideration clearly shows that fluctuations of density such as vacancies (holes, cavities) in diverse solvents should be taken into account at evaluations solubility of different solutes. This fact leads to paradoxy at the first glance conclusion that the excess volume plays a very modest role in microscopic balance of volumes at dissolution process, at least, in considered cases.

The calculated values of solubility based on values of cavities obtained from spectral shifts data are close enough to empirical ones, and evaluated size 
Table 3. Geometrical characteristics and quasi-chemical potentials of dissolution of some alkyl substituted benzenes in water at $293 \mathrm{~K}$ and solute contractions.

\begin{tabular}{|c|c|c|c|c|c|c|c|c|}
\hline \multirow{3}{*}{ Solute } & \multirow{3}{*}{$10^{10} r, \mathrm{~m}$} & \multirow{3}{*}{$\Delta N_{1}$} & \multicolumn{5}{|c|}{$\mu / k T$} & \multirow{3}{*}{$10^{10} \delta r . \mathrm{m}$} \\
\hline & & & \multicolumn{4}{|c|}{ Calculation } & \multirow[b]{2}{*}{ Experiment } & \\
\hline & & & Equation (16) & Equation (24) & Equation (32) & $\begin{array}{c}\text { With Equation } \\
\text { (24) }\end{array}$ & & \\
\hline$\alpha$-methylstyrene & 3.145 & 9.6 & 13.30 & 11.27 & 12.29 & 11.47 & - & -0.07 \\
\hline mesitylene & 3.19 & 10.7 & 13.94 & 11.20 & 12.57 & 11.67 & $11.47 ; 12.72$ & -0.10 \\
\hline pseudo-cumene & 3.19 & 10.7 & 13.94 & 11.10 & 12.52 & 11.62 & - & -0.10 \\
\hline hemellitene & 3.19 & 10.7 & 13.94 & 11.72 & 12.83 & 11.93 & $1152 ; 11.83$ & -0.08 \\
\hline$o$-ethyltoluene & 3.19 & 10.7 & 13.94 & 11.21 & 12.58 & 11.68 & - & -0.09 \\
\hline m-ethyltoluene & 3.19 & 10.7 & 13.94 & 11.01 & 12.48 & 11.58 & - & -0.10 \\
\hline$\Pi$-etyltoluene & 3.19 & 10.7 & 13.94 & 11.00 & 12.47 & 11.57 & - & -0.10 \\
\hline
\end{tabular}

changes, and change of strength of hydrogen bonds in the solvent near the solutes look right. So, the main points of the above consideration are correct.

In essence, the called conclusions are obtained owing to taking into account, evidently or not evidently, effect of electric field described in ref. [9]. Hence such effect should be taken into consideration in advanced models of solubility, including computer simulation.

\section{Conflicts of Interest}

The author declares no conflicts of interest regarding the publication of this paper.

\section{References}

[1] Hildebrand, J.H. (1916) Solubility. Journal of the American Chemical Society, 38, 1452-1473. https://doi.org/10.1021/ja02265a002

[2] Hansen, C.M. (2000) Hansen Solubility Parameters. A User's Handbook. CRC Press, Boca Raton, London, New York, Washington DC.

[3] Ben-Naim, A. (2006) Molecular Theory of Solutions. Oxford University Press, Oxford.

[4] Graziano, G.J. (1998) On the Size Dependence of Hydrophobic Hydration. Journal of the Chemical Society, Faraday Transactions, 94, 3345-3352.

https://doi.org/10.1039/a805733h

[5] Frenkel, Y.I. (1975) Kinetic Theory of Liquids. Nauka, Leningrad.

[6] Luck, W.A.P. (1981) Einfache Modelle für kalorische Eigenschaften der Flüssigkeiten. Berichte der Bunsengesellschaft für physikalische Chemie, 83, 859.

[7] Dejardin, L.J., Marrony, R., Delseny, C., Brunet, S. and Berge, R. (1981) ne nouvelle définition des volumes libres dans les liquides non associés: Application à la détermination des diamètres moléculaires. Rheologica Acta, 20, 497-500. https://doi.org/10.1007/BF01503272

[8] Ar'ev, I.A. (1987) Investigation the benzene hydration with spectral shift method. Theoretical and Experimental Chemistry, 23, 329. 
[9] Ar'ev, I.A. and Chernova, L.G. (2011) Interaction of Benzene with Water and Aqueous Solutions of Alkali Metal Chlorides. Russian Journal of Physical Chemistry $A, 85,1592$. https://doi.org/10.1134/S0036024411090032

[10] Ar'ev, I.A., Dyadyusha, G.G. and Makhlinets, N.V. (1983) Effect of Spin-Orbital Interaction in Mono- and p-Dihalogenobezene Molecule on Their Electronic Spectrum Shifts under Solvent Action. Optics and Spectroscopy, 55, 285.

[11] Ar'ev, I.A., Dyadyusha, G.G., Klimusheva, G.V. and Soroka, G.M. (1983) Effect of Spin-Orbital Interaction in Mono- and p-Dihalogenobenzenes in the S1 State under the Action of Environment. Optics and Spectroscopy, 55, 653.

[12] Ar'ev, I.A., Lebovka, N.I. and Solovieva, E.A. (2013) Effects of Partial Charge-Transfer Solute-Solvent Interactions in Absorption Spectra of Aromatic Hydrocarbons in Aqueous and Alcoholic Solutions. Molecular Physics, 111, 3077-3080. https://doi.org/10.1080/00268976.2013.770175

[13] Sinanoglu, O. (1981) Microscopic Surface Tension down to Molecular Dimensions and Microthermodynamic Surface Areas of Molecules or Clusters. The Journal of Chemical Physics, 75, 463. https://doi.org/10.1063/1.441807

[14] Sinanoglu, O. (1981) What Size Cluster Is Like a Surface? Chemical Physics Letters, 81, 188-190. https://doi.org/10.1016/0009-2614(81)80233-3

[15] Ar'ev, I.A. (2014) Correcting the Microscopic Coefficient of Surface Tension of Associated Liquids. Russian Journal of Physical Chemistry A, 88, 173-174. https://doi.org/10.1134/S003602441401004X

[16] Vargaftik, N.B., Volyak, L.D. and Volkov, B.N. (1975) Surface Tension of Water at Temperatures from 0 up to 370 Degrees of Celsium. In: Surface Phenomena in Liquids, Leningrad University, Leningrad, 180-192. (In Russian)

[17] Zhang, X. and Gobas, F.A.P.C. (1995) A Thermodynamic Analysis of the Relationships between Molecular Size, Hydrophobicity, Aqueous Solubility and Octanol=Water Partitioning of Organic Chemicals. Chemosphere, 31, 3501.

[18] Nikolskii, B.P. (1966) Handbook for Chemist. Vol. 1, 2, Khimia, Moscow, Leningrad. (In Russian)

[19] Samoylov, O.Ya. (1957) The Structure of Aqueous Solutions of Electrolytes and Hydration of Ions. Acad. Sci. of USSR, Moscow. (In Russian) 